\title{
ON POWER BOUNDED OPERATORS WITH HOLOMORPHIC EIGENVECTORS, II
}

\author{
MARIA F. GAMAL'
}

\begin{abstract}
In [U] (among other results), M. Uchiyama gave the necessary and sufficient conditions for contractions to be similar to the unilateral shift $S$ of multiplicity 1 in terms of norm-estimates of complete analytic families of eigenvectors of their adjoints. In [G2], it was shown that this result for contractions can't be extended to power bounded operators. Namely, a cyclic power bounded operator was constructed which has the requested norm-estimates, is a quasiaffine transform of $S$, but is not quasisimilar to $S$. In this paper, it is shown that the additional assumption on a power bounded operator to be quasisimilar to $S$ (with the requested norm-estimates) does not imply similarity to $S$. A question whether the criterion for contractions to be similar to $S$ can be generalized to polynomially bounded operators remains open.

Also, for every cardinal number $2 \leq N \leq \infty$ a power bounded operator $T$ is constructed such that $T$ is a quasiaffine transform of $S$ and $\operatorname{dim} \operatorname{ker} T^{*}=N$. This is impossible for polynomially bounded operators. Moreover, the constructed operators $T$ have the requested normestimates of complete analytic families of eigenvectors of $T^{*}$.
\end{abstract}

\section{INTRODUCTION}

Let $\mathcal{H}$ be a (complex, separable) Hilbert space, and let $T$ be a (linear, bounded) operator acting on $\mathcal{H}$. The operator $T$ is called power bounded, if $\sup _{n \geq 1}\left\|T^{n}\right\|<\infty$. The operator $T$ is called polynomially bounded, if there exists a constant $C>0$ such that $\|p(T)\| \leq C \max \{|p(z)|,|z| \leq 1\}$ for every (analytic) polinomial $p$. The operator $T$ is a contraction if $\|T\| \leq 1$. It is well known that a contraction is polynomially bounded, and, consequently, power bounded.

Let $T$ and $R$ be operators on spaces $\mathcal{H}$ and $\mathcal{K}$, respectively, and let $X$ : $\mathcal{H} \rightarrow \mathcal{K}$ be a (linear, bounded) transformation such that $X$ intertwines $T$ and $R$, that is, $X T=R X$. If $X$ is unitary, then $T$ and $R$ are called unitarily equivalent, in notation: $T \cong R$. If $X$ is invertible (that is, its inverse is bounded), then $T$ and $R$ are called similar, in notation: $T \approx R$. If $X$ is a quasiaffinity, that is, $\operatorname{ker} X=\{0\}$ and $\operatorname{clos} X \mathcal{H}=\mathcal{K}$, then $T$ is called a quasiaffine transform of $R$, in notation: $T \prec R$. If $T \prec R$ and $R \prec T$, then $T$ and $R$ are called quasisimilar, in notation: $T \sim R$.

In [U], necessary and sufficient conditions for contractions to be quasiaffine transforms, quasisimilar, or similar to unilateral shifts of finite multiplicity in terms of norm-estimates of complete analytic families of eigenvectors of their adjoints are given. In [G2], the result from [U] for contractions to be

2010 Mathematics Subject Classification. Primary 47A05, 47B99, 47B32, 30H10.

Key words and phrases. Power bounded operator, unilateral shift, similarity, quasisimilarity, quasiaffine transform, analytic family of eigenvectors. 
quasiaffine transforms of unilateral shifts of finite multiplicity is generalized to power bounded operators. Also, in [G2] an example of a cyclic power bounded operator $T_{0}$ is given such that $T_{0}$ satisfies sufficient conditions on contractions to be similar to the unilateral shift $S$ of multiplicity 1, but there is no contraction $R$ such that $R \prec T$. This example is based on some example from [MT].

In this paper, it is shown that the additional assumption $T \sim S$ on a power bounded operator $T$ (with the requested norm-estimates of complete analytic family of eigenvectors of $T^{*}$ ) also does not imply the similarity $T \approx S$. The constructed operator $T$ has the following property: there exist two invariant subspaces $\mathcal{M}_{1}, \mathcal{M}_{2}$ of $T$ such that $\left.T\right|_{\mathcal{M}_{k}} \approx S(k=1,2)$ and $\mathcal{M}_{1} \vee \mathcal{M}_{2}$ is the whole space on which $T$ acts. The same property takes place for polynomially bounded operators that are quasiaffine transforms of $S$ [G3]. A question whether the criterion for contractions to be similar to $S$ can be generalized to polynomially bounded operators remains open.

If a polynomially bounded operator $T$ is such that $T \prec S$, then the range of $T$ is closed and $\operatorname{dim} \operatorname{ker} T^{*}=1$ ([T], [BP], [G1], see Remark 3.3 below). The range of a cyclic power bounded operator $T_{0}$ constructed in [G2] is not closed. It allows, for every cardinal number $2 \leq N \leq \infty$, to construct example of a power bounded operator $T$ such that $T \prec S$, a complete analytic family of eigenvectors of $T^{*}$ has the requested norm-estimates, and $\operatorname{dim} \operatorname{ker} T^{*}=N$.

The paper is organized as follows. In Sec. 2 a power bounded operator $T$ is constructed such that $T \sim S$, a complete analytic family of eigenvectors of $T^{*}$ has the requested norm-estimates, but $T \not \approx S$. In Sec. 3 it is shown that if a power bounded operator $T$ is such that $T \prec S$, then $\operatorname{dim} \operatorname{ker} T^{*}$ can be arbitrarily large. In Sec. 4 it is shown that for $T_{0}$ constructed in [G2] $\sigma_{e}\left(T_{0}\right)$ is the closed unit disc.

The following notation will be used. Let $\mathcal{H}$ be a Hilbert space, and let $\mathcal{M}$ be its (linear, closed) subspace. By $I_{\mathcal{H}}$ and $P_{\mathcal{M}}$ the identical operator on $\mathcal{H}$ and the orthogonal projection from $\mathcal{H}$ onto $\mathcal{M}$ are denoted, respectively. For an operator $T: \mathcal{H} \rightarrow \mathcal{H}$, a subspace $\mathcal{M}$ of $\mathcal{H}$ is called invariant subspace of $T$, if $T \mathcal{M} \subset \mathcal{M}$. The complete lattice of all invariant subspaces of $T$ is denoted by Lat $T$.

The words "operator" and "transformation" mean that the linear mappings under consideration are bounded.

The symbols $\mathbb{D}, \mathbb{T}$, and $m$ denote the open unit disc, the unit circle, and the normalized Lebesgue measure on $\mathbb{T}$, respectively. $H^{\infty}$ is the Banach algebra of all analytic bounded functions in $\mathbb{D}$. $H^{2}$ is the Hardy space on $\mathbb{D}, S: H^{2} \rightarrow H^{2},(S h)(z)=z h(z)\left(z \in \mathbb{D}, h \in H^{2}\right)$ is the unilateral shift of multiplicity 1 . Set

$$
h_{\lambda}(z)=\frac{1}{1-\bar{\lambda} z}, \quad z, \lambda \in \mathbb{D} .
$$

It is well known and easy to see that

$$
S^{*} h_{\lambda}=\bar{\lambda} h_{\lambda}, \quad\left\|h_{\lambda}\right\|=\frac{1}{\left(1-|\lambda|^{2}\right)^{1 / 2}} \quad(\lambda \in \mathbb{D}),
$$

and the function $\mathbb{D} \rightarrow H^{2}, \lambda \mapsto h_{\lambda}$, is conjugate analytic. 
If $T$ is an operator on a Hilbert space $\mathcal{H}$ and a quasiaffinity $X$ is such that $X T=S X$, then

$$
T^{*} X^{*} h_{\lambda}=\bar{\lambda} X^{*} h_{\lambda}, \quad\left\|X^{*} h_{\lambda}\right\| \leq\|X\| \frac{1}{\left(1-|\lambda|^{2}\right)^{1 / 2}} \quad(\lambda \in \mathbb{D}),
$$

and the function $\mathbb{D} \rightarrow \mathcal{H}, \lambda \mapsto X^{*} h_{\lambda}$, is conjugate analytic. If, in addition, $T$ is a contraction and $\left\|X^{*} h_{\lambda}\right\| \asymp \frac{1}{\left(1-|\lambda|^{2}\right)^{1 / 2}}$, then, by [U], $T \approx S$.

\section{EXAMPlE OF OPERATOR QUASISIMILAR TO $S$}

In this section a power bounded operator $T$ is constructed such that $T \sim S$, a complete analytic family of eigenvectors $k_{\lambda}$ of $T^{*}$ has the estimate $\left\|k_{\lambda}\right\| \asymp\left\|h_{\lambda}\right\|$, but $T$ is not polynomially bounded. Consequently, $T \not \approx S$.

For an inner function $\theta$ set $\mathcal{K}_{\theta}=H^{2} \ominus \theta H^{2}, T_{\theta}=\left.P_{\mathcal{K}_{\theta}} S\right|_{\mathcal{K}_{\theta}}$, and

$$
h_{\theta, \lambda}(z)=\frac{1-\overline{\theta(\lambda)} \theta(z)}{1-\bar{\lambda} z}, \quad z, \lambda \in \mathbb{D} .
$$

It is well known and easy to see that

$$
P_{\theta H^{2}} h_{\lambda}=\overline{\theta(\lambda)} \theta h_{\lambda} \quad \text { and } \quad P_{\mathcal{K}_{\theta}} h_{\lambda}=h_{\theta, \lambda} .
$$

Furthermore,

$$
f(\lambda)=\left(f, h_{\theta, \lambda}\right) \text { for every } \lambda \in \mathbb{D} \text { and } f \in \mathcal{K}_{\theta} .
$$

2.1. Bases of a Hilbert space. In this subsection we recall the notions and properties of (not orthogonal) bases of a Hilbert space. For references, see [N1, Ch. VI.3] or [N2, Ch. I.A.5.1, I.A.5.6.2, II.C.3.1].

Let $\mathcal{H}$ be a Hilbert space, and let $\left\{x_{n}\right\}_{n \geq 0} \subset \mathcal{H}$. The family $\left\{x_{n}\right\}_{n \geq 0}$ is called an unconditional basis of $\mathcal{H}$, if for every $x \in \mathcal{H}$ there exists a family $\left\{a_{n}\right\}_{n \geq 0} \subset \mathbb{C}$ such that $x=\sum_{n \geq 0} a_{n} x_{n}$ and the series $\sum_{n \geq 0} a_{n} x_{n}$ converges unconditionally, that is, for every $\varepsilon>0$ there exists a finite $\mathfrak{N} \subset\{0,1, \ldots\}$ such that $\left\|x-\sum_{n \in \mathfrak{N}^{\prime}} a_{n} x_{n}\right\|<\varepsilon$ for every finite $\mathfrak{N} \subset \mathfrak{N}^{\prime} \subset\{0,1, \ldots\}$. The family $\left\{x_{n}\right\}_{n>0}$ is called a Riesz basis of $\mathcal{H}$, if the mapping $W$ acting by the formula $W e_{n}=x_{n}$ for an orthonormal basis $\left\{e_{n}\right\}_{n>0}$ is an invertible transformation. Let $\left\{x_{n}\right\}_{n>0}$ is such that $\mathcal{H}=\vee_{n \geq 0} x_{n}, x_{n} \notin \vee_{k \neq n} x_{k}$ for all $n$, and $\left\|x_{n}\right\| \asymp 1$. Then $\left\{x_{n}\right\}_{n \geq 0}$ is an unconditional basis if and only if $\left\{x_{n}\right\}_{n \geq 0}$ is a Riesz basis.

Let $\mathcal{H}=\vee_{n \geq 0} x_{n}$, and let $\left\{x_{n}\right\}_{n=0}^{N}$ are linear independent for every finite $N$. Define mappings $\mathcal{Q}_{n}$ and $\mathcal{P}_{n}$ on the linear set

by the formulas

$$
\left\{\sum_{n=0}^{N} a_{n} x_{n},\left\{a_{n}\right\}_{n \geq 0} \subset \mathbb{C}, N=0,1, \ldots\right\}
$$

$$
\mathcal{Q}_{n} \sum_{k \geq 0} a_{k} x_{k}=a_{n} x_{n}
$$

and

$$
\mathcal{P}_{n} \sum_{k \geq 0} a_{k} x_{k}=\sum_{k=0}^{n} a_{k} x_{k} .
$$

Clearly, $\mathcal{Q}_{n}=\mathcal{P}_{n}-\mathcal{P}_{n-1}$ for all $n \geq 1$, and $\mathcal{Q}_{0}=\mathcal{P}_{0}$. 
Let $n \geq 0$. Then the following are equivalent: (i) $\mathcal{Q}_{n}$ can be extended on $\mathcal{H}$ as an operator; (ii) there exists $x_{n}^{\prime} \in \mathcal{H}$ such that $\left(x_{n}^{\prime}, x_{n}\right)=1$ and $\left(x_{n}^{\prime}, x_{k}\right)=0$, if $k \neq n$; (iii) $x_{n} \notin \vee_{k \neq n} x_{k}$.

If $\mathcal{P}_{n}$ can be extended on $\mathcal{H}$ as operators for all $n$ and

$$
\sup _{n \geq 0}\left\|\mathcal{P}_{n}\right\|<\infty
$$

then $\mathcal{P}_{n} x=\sum_{k=0}^{n}\left(x, x_{k}^{\prime}\right) x_{k}$ for all $n$ and

$$
x=\lim _{n} \mathcal{P}_{n} x=\sum_{k \geq 0}\left(x, x_{k}^{\prime}\right) x_{k}\left(=\lim _{n} \sum_{k=0}^{n}\left(x, x_{k}^{\prime}\right) x_{k}\right) \quad \text { for every } x \in \mathcal{H} .
$$

Lemma 2.1. Suppose that $\mathcal{H}$ and $\mathcal{K}$ are Hilbert spaces, $\mathcal{H}=\vee_{n>0} x_{n}$, $\mathcal{K}=\vee_{n \geq 0} y_{n}, \mathcal{Q}_{n}$ and $\mathcal{P}_{n}$ defined by (2.4) and (2.5) for $\left\{y_{n}\right\}_{n \geq 0}$ and $\left\{x_{n}\right\}_{n \geq 0}$, respectively, are operators for all $n$, and (2.6) is fulfilled for $\mathcal{P}_{n}$. Furthermore, suppose that the family $\left\{c_{n}\right\}_{n \geq 0} \subset \mathbb{C}$ is such that $c_{n} \neq 0$ for every $n \geq 0$ and the mapping $X$ acting by the formula $X x_{n}=c_{n} y_{n}, n \geq 0$, is a transformation. Then $X: \mathcal{H} \rightarrow \mathcal{K}$ is a quasiaffinity.

Proof. Since $y_{n} \in X \mathcal{H}$ and $\mathcal{K}=\vee_{n \geq 0} y_{n}$, we conclude that $\operatorname{clos} X \mathcal{H}=\mathcal{K}$. Let $x \in \mathcal{H}$ be such that $X x=0$. We have $x=\lim _{N} \sum_{k=0}^{N} a_{k} x_{k}$ (where $\left.a_{k}=\left(x, x_{k}^{\prime}\right)\right)$, therefore,

$$
0=\mathcal{Q}_{n} X x=\mathcal{Q}_{n} X\left(\lim _{N} \sum_{k=0}^{N} a_{k} x_{k}\right)=\lim _{N} \mathcal{Q}_{n} \sum_{k=0}^{N} a_{k} c_{k} y_{k}=a_{n} c_{n} y_{n}
$$

for every $n \geq 0$. Since $c_{n} \neq 0$, we conclude that $a_{n}=0$ for every $n \geq 0$. Therefore, $x=0$.

Lemma 2.2. Suppose that $\mathcal{H}$ and $\mathcal{K}$ are Hilbert spaces, $\left\{u_{n}\right\}_{n>0}$ is a Riesz basis of $\mathcal{K}$, the family $\left\{x_{n}\right\}_{n \geq 0} \subset \mathcal{H}$ is such that

$$
\mathcal{H}=\bigvee_{n \geq 0} x_{n}, \quad \inf _{n \geq 0}\left\|x_{n}\right\|>0
$$

and $\mathcal{Q}_{n}$ defined by $(2.4)$ are such that

$$
\sup _{n \geq 0}\left\|\mathcal{Q}_{n}\right\|<\infty .
$$

Let $\left\{c_{n}\right\}_{n \geq 0} \subset \mathbb{C}$ be such that $\sum_{n \geq 0}\left|c_{n}\right|^{2}<\infty$. Then the mapping $Z: \mathcal{H} \rightarrow$ $\mathcal{K}$ acting by the formula $Z x_{n}=c_{n} u_{n}, n \geq 0$, is a transformation.

Proof. Since $\left\{u_{n}\right\}_{n \geq 0}$ is a Riesz basis,

$$
\begin{aligned}
\left\|Z \sum_{n \geq 0} a_{n} x_{n}\right\|^{2} & =\left\|\sum_{n \geq 0} c_{n} a_{n} u_{n}\right\|^{2} \asymp \sum_{n \geq 0}\left|c_{n}\right|^{2}\left|a_{n}\right|^{2} \\
& \leq \frac{1}{\left(\inf _{n \geq 0}\left\|x_{n}\right\|\right)^{2}} \sum_{n \geq 0}\left|c_{n}\right|^{2}\left\|\mathcal{Q}_{n} \sum_{k \geq 0} a_{k} x_{k}\right\|^{2} \\
& \leq \frac{\left(\sup _{n \geq 0}\left\|\mathcal{Q}_{n}\right\|\right)^{2}}{\left(\inf _{n \geq 0}\left\|x_{n}\right\|\right)^{2}} \sum_{n \geq 0}\left|c_{n}\right|^{2}\left\|\sum_{k \geq 0} a_{k} x_{k}\right\|^{2}
\end{aligned}
$$


In Lemma 2.3 the notion of a Helson-Szegö weight function is used. To the definition of this notion we refer to the references in Lemma 2.3. This notion will not be used in the sequel.

Lemma 2.3 ([BS, Theorem 2.1], [N1, Ch. VIII.6], [N2, Lemma I.A.5.2.5, Theorem I.A.5.4.1]). Suppose that $\psi \in H^{\infty}$ is an outer function, $|\psi|^{2}$ is a Helson-Szegö weight function, and $1 / \psi \notin H^{\infty}$. Set $\mathcal{H}_{0}=H^{2}$ and $x_{n}=\chi^{n} \psi$, $n \geq 0$, where $\chi(\zeta)=\zeta, \zeta \in \mathbb{T}$. Then $\mathcal{H}_{0}$ and $\left\{x_{n}\right\}_{n \geq 0}$ satisfy $(2.7),\left\{\mathcal{P}_{n}\right\}_{n \geq 0}$ defined by (2.5) satisfies (2.6), for every Riesz basis $\left\{u_{n}\right\}_{n \geq 0}$ of a Hilbert space $\mathcal{K}$ the mapping $W: \mathcal{K} \rightarrow \mathcal{H}_{0}$ acting by the formula $W u_{n}=x_{n}, n \geq 0$, is a transformation, but $\left\{x_{n}\right\}_{n \geq 0}$ is not a Riesz basis of $\mathcal{H}_{0}$.

Proof. Clearly, $\left\{\chi^{n}\right\}_{n \geq 0}$ is an orthonormal basis of $H^{2}$. Define $J: H^{2} \rightarrow H^{2}$ by the formula $J h=\psi h, h \in H^{2}$. Clearly, $J \chi^{n}=x_{n}, n \geq 0$, and $J$ is an operator, because $\psi \in H^{\infty}$. Thus, the statement about $W$ is proved.

If we assume that $\left\{x_{n}\right\}_{n \geq 0}$ is a Riesz basis of $H^{2}$, then the operator $J$ must have bounded inverse on $H^{2}$. Since $1 / \psi \notin H^{\infty}$, we conclude that $J$ has no bounded inverse.

All remaining statements follow from the references.

Example 2.4 ([BS, Example 3.3.2], [N2, Ch. I.A.5.5]). Let $0<\alpha<1 / 2$. Set $\psi(z)=(1-z)^{\alpha}, z \in \mathbb{D}$. Then $\psi$ satisfies Lemma 2.3 .

Recall that an operator $T$ satisfies the Tadmor-Ritt condition, if there exists $C>0$ such that $\left\|(T-z I)^{-1}\right\| \leq C /|z-1|$ for $z \in \mathbb{C},|z|>1$. The Tadmor-Ritt condition implies power boundedness [L], [NZ], [V2]. The Tadmor-Ritt condition will not be used in the sequel.

Lemma 2.5 ([V1, Lemma 2.2]). Suppose that $\left\{\lambda_{n}\right\}_{n \geq 0} \subset(0,1), \lambda_{n}<\lambda_{n+1}$ for all $n$, and $\lambda_{n} \rightarrow 1$. Furthermore, suppose that $\mathcal{H}_{0}$ is a Hilbert space, $\mathcal{H}_{0}=\vee_{n \geq 0} x_{n}, \mathcal{P}_{n}: \mathcal{H}_{0} \rightarrow \mathcal{H}_{0}$ defined by (2.5) are operators, and (2.6) is fulfilled. Then the mapping $R_{0}: \mathcal{H}_{0} \rightarrow \mathcal{H}_{0}$ acting by the formula

$$
R_{0} x_{n}=\lambda_{n} x_{n}, \quad n \geq 0,
$$

is an operator, $R_{0}$ satisfies the Tadmor-Ritt condition and, consequently, is power bounded.

2.2. Blaschke product. In this subsection we recall the well-known facts about Blaschke products which will be used in the sequel. For references, see [N1, Ch. VI.2, IX.3] or [N2, Ch. II.C.3.2, Lemma II.C.3.2.18].

For $\lambda \in \mathbb{D}$, a Blaschke factor is $b_{\lambda}(z)=\frac{|\lambda|}{\lambda} \frac{\lambda-z}{1-\bar{\lambda} z}, z \in \mathbb{D}$. The following equality will be used:

$$
1-\left|b_{\lambda}(z)\right|^{2}=\frac{\left(1-|z|^{2}\right)\left(1-|\lambda|^{2}\right)}{|1-\bar{\lambda} z|^{2}} .
$$

If $\left\{\lambda_{n}\right\}_{n} \subset \mathbb{D}$ satisfies the Blaschke condition $\sum_{n}\left(1-\left|\lambda_{n}\right|\right)<\infty$, then the Blaschke product $B=\prod_{n} b_{\lambda_{n}}$ converges and $B$ is an inner function.

Let $B=\prod_{n} b_{\lambda_{n}}$ be a Blaschke product with simple zeros, that is, $\lambda_{n} \neq \lambda_{k}$, if $n \neq k$. Set $B_{n}=\prod_{k \neq n} b_{\lambda_{k}}$,

$$
u_{n}=\left(1-\left|\lambda_{n}\right|^{2}\right)^{1 / 2} h_{\lambda_{n}}, \quad v_{n}=\frac{1}{B_{n}\left(\lambda_{n}\right)} B_{n} u_{n},
$$


where $h_{\lambda}$ are defined in (1.1), then

$$
\begin{aligned}
\left\|u_{n}\right\|=1,\left\|v_{n}\right\| & =\frac{1}{\left|B_{n}\left(\lambda_{n}\right)\right|}, \quad\left(v_{n}, u_{n}\right)=1, \quad\left(v_{n}, u_{k}\right)=0, \quad \text { if } n \neq k, \\
\mathcal{K}_{B} & =\vee_{n} u_{n}=\vee_{n} v_{n}, \quad \text { and } \quad T_{B}^{*} u_{n}=\overline{\lambda_{n}} u_{n} .
\end{aligned}
$$

Let $\left\{\lambda_{n}\right\}_{n} \subset \mathbb{D}$ be such that $\lambda_{n} \neq \lambda_{k}$, if $n \neq k$. The family $\left\{\lambda_{n}\right\}_{n}$ satisfies the Carleson interpolating condition (the Carleson condition for brevity), if

$$
\text { there exists } \delta>0 \text { such that }\left|B_{n}\left(\lambda_{n}\right)\right| \geq \delta \text { for every } n \text {. }
$$

Then $\left\{u_{n}\right\}_{n}$ and $\left\{v_{n}\right\}_{n}$ are Riesz bases of $\mathcal{K}_{B}$, and

$$
f=\sum_{n}\left(f, v_{n}\right) u_{n} \quad \text { for every } f \in \mathcal{K}_{B}
$$

Set

$$
D_{n}=\left\{z \in \mathbb{D}:\left|b_{\lambda_{n}}(z)\right| \leq \delta / 3\right\}
$$

Then

$$
\left|B_{n}(z)\right| \geq \delta / 2, \quad \text { if } z \in D_{n}
$$

and

$$
|B(z)| \geq \delta^{2} / 6, \quad \text { if } z \in \mathbb{D} \backslash \cup_{n} D_{n} .
$$

Lemma 2.6. Suppose that $\left\{\lambda_{n}\right\}_{n>0} \subset \mathbb{D}, \lambda_{n} \neq \lambda_{k}$, if $n \neq k$, and $\left\{\lambda_{n}\right\}_{n>0}$ satisfies the Carleson condition (2.11). Furthermore, suppose that $\mathcal{H}_{0}$ is a Hilbert space, $\left\{x_{n}\right\}_{n \geq 0} \subset \mathcal{H}_{0}$ satisfies $(2.7), \mathcal{Q}_{n}: \mathcal{H}_{0} \rightarrow \mathcal{H}_{0}$ acting by the formula (2.4) are operators and (2.8) is fulfilled. Finally, suppose that

$W: \mathcal{K}_{B} \rightarrow \mathcal{H}_{0}$ is a transformation and $x_{n}=W u_{n}$ for all $n \geq 0$, where $u_{n}$ are defined in (2.10). Then

$\left\|W h_{B, \lambda}\right\| \geq \frac{\delta}{2}\left(1-\frac{\delta^{2}}{9}\right)^{1 / 2} \frac{\inf _{n \geq 0}\left\|x_{n}\right\|}{\sup _{n \geq 0}\left\|\mathcal{Q}_{n}\right\|} \frac{1}{\left(1-|\lambda|^{2}\right)^{1 / 2}} \quad$ for every $\lambda \in \cup_{n \geq 0} D_{n}$, where $h_{B, \lambda}$ are defined in (2.1), $\delta$ is from (2.11) and $D_{n}$ are defined in (2.13).

Proof. By (2.12), $h_{B, \lambda}=\sum_{k \geq 0}\left(h_{B, \lambda}, v_{k}\right) u_{k}$. Therefore,

$$
W h_{B, \lambda}=\sum_{k \geq 0}\left(h_{B, \lambda}, v_{k}\right) x_{k}
$$

We have

$$
\left\|W h_{B, \lambda}\right\| \geq \frac{1}{\sup _{k}\left\|\mathcal{Q}_{k}\right\|}\left\|\mathcal{Q}_{n} W h_{B, \lambda}\right\|=\frac{1}{\sup _{k}\left\|\mathcal{Q}_{k}\right\|}\left|\left(h_{B, \lambda}, v_{n}\right)\right|\left\|x_{n}\right\| .
$$

By (2.3), (2.10), and (2.14),

$$
\begin{aligned}
\left|\left(h_{B, \lambda}, v_{n}\right)\right| & =\left|v_{n}(\lambda)\right|=\frac{\left|B_{n}(\lambda)\right|}{\left|B_{n}\left(\lambda_{n}\right)\right|} \frac{\left(1-\left|\lambda_{n}\right|^{2}\right)^{1 / 2}}{\left|1-\bar{\lambda}_{n} \lambda\right|} \\
& \geq \frac{\delta}{2} \frac{\left(1-|\lambda|^{2}\right)^{1 / 2}\left(1-\left|\lambda_{n}\right|^{2}\right)^{1 / 2}}{\left|1-\bar{\lambda}_{n} \lambda\right|} \frac{1}{\left(1-|\lambda|^{2}\right)^{1 / 2}}, \quad \lambda \in D_{n} .
\end{aligned}
$$


By (2.9) and (2.13),

$$
\frac{\left(1-|\lambda|^{2}\right)^{1 / 2}\left(1-\left|\lambda_{n}\right|^{2}\right)^{1 / 2}}{\left|1-\bar{\lambda}_{n} \lambda\right|}=\left(1-\left|b_{\lambda_{n}}(\lambda)\right|^{2}\right)^{1 / 2} \geq\left(1-\frac{\delta^{2}}{9}\right)^{1 / 2}, \quad \lambda \in D_{n} .
$$

2.3. Construction of example. The following lemma is a corollary of Sec. 2.1 and 2.2 .

Lemma 2.7. Suppose that $\left\{\lambda_{n}\right\}_{n \geq 0}, \mathcal{H}_{0}$ and $\left\{x_{n}\right\}_{n \geq 0}$ satisfy assumption of Lemma 2.5, $\left\{\lambda_{n}\right\}_{n \geq 0}$ satisfies the Carleson condition (2.11), and $\left\{x_{n}\right\}_{n \geq 0}$ satisfies (2.7). Let $R_{0}$ be the operator from Lemma 2.5. Let $g \in H^{\infty}$ be such that

$$
\sum_{n \geq 0}\left|g\left(\lambda_{n}\right)\right|^{2}<\infty
$$

Set $c_{n}=g\left(\lambda_{n}\right), n \geq 0$. Define $Z$ as in Lemma 2.2. Suppose that the mapping $W: \mathcal{K}_{B} \rightarrow \mathcal{H}_{0}$ acting by the formula $W u_{n}=x_{n}, n \geq 0$, is a transformation. Then $W T_{B}^{*}=R_{0} W, Z R_{0}=T_{B}^{*} Z$, and $Z W=g\left(T_{B}^{*}\right)$.

Proof. Set $\mathcal{Q}_{n}=\mathcal{P}_{n}-\mathcal{P}_{n-1}, n \geq 1$, and $\mathcal{Q}_{0}=\mathcal{P}_{0}$. Clearly, $\mathcal{Q}_{n}$ act by the formula (2.4), and (2.8) is fulfilled. The lemma follows from Lemma 2.2 and the definitions of $W, Z$, and $R_{0}$.

Example 2.8. Suppose that $\left\{\lambda_{n}\right\}_{n>0} \subset(0,1)$ satisfies the Blaschke condition, $\lambda_{n} \neq \lambda_{k}$, if $n \neq k$, and $g(z)=(1-z)^{1 / 2}, z \in \mathbb{D}$. Then $g$ is outer, $g$ satisfies (2.16) and $\sum_{n \geq 0}|\widehat{g}(n)|<\infty$ by [D, Corollary of Theorem 3.15], because $g^{\prime} \in H^{1}$.

The following theorem is the main result of Sec. 2 .

Theorem 2.9. Suppose that $\left\{\lambda_{n}\right\}_{n \geq 0} \subset(0,1), \lambda_{n}<\lambda_{n+1}$ for all $n$, and $\left\{\lambda_{n}\right\}_{n \geq 0}$ satisfies the Carleson condition (2.11). Suppose that $\mathcal{H}_{0}$ and $\left\{x_{n}\right\}_{n \geq 0}$ satisfy the assumptions of Lemmas 2.5 and 2.6, but $\left\{x_{n}\right\}_{n \geq 0}$ is not a Riezs basis of $\mathcal{H}_{0}$. Suppose that $g \in H^{\infty}$ is outer, and $g$ satisfies (2.16). Set $g_{*}(z)=\overline{g(\bar{z})}, z \in \mathbb{D}$. Finally, suppose that $R_{0}, W, Z$ are from Lemmas 2.5, 2.6, and 2.7, respectively. Set

$$
\begin{gathered}
T: B H^{2} \oplus \mathcal{H}_{0} \rightarrow B H^{2} \oplus \mathcal{H}_{0}, \quad Y: B H^{2} \oplus \mathcal{H}_{0} \rightarrow H^{2}, X: H^{2} \rightarrow B H^{2} \oplus \mathcal{H}_{0}, \\
T=\left(\begin{array}{cc}
\left.S\right|_{B H^{2}} & \left.P_{B H^{2}} S\right|_{\mathcal{K}_{B}} W^{*} \\
\mathbb{O} & R_{0}^{*}
\end{array}\right), \\
Y=\left(\begin{array}{cc}
I_{B H^{2}} & \mathbb{O} \\
\mathbb{O} & W^{*}
\end{array}\right), \quad X=\left(\begin{array}{cc}
\left.g_{*}(S)\right|_{B H^{2}} & \left.P_{B H^{2}} g_{*}(S)\right|_{\mathcal{K}_{B}} \\
\mathbb{O} & Z^{*}
\end{array}\right) .
\end{gathered}
$$

Then $T$ is power bounded, $T$ is not polynomially bounded, $X$ and $Y$ are quasiaffinities, $Y T=S Y, X S=T X, Y X=g_{*}(S)$, and if $\sum_{n \geq 0}|\widehat{g}(n)|<$ $\infty$, then $X Y=g_{*}(T)$. Moreover,

$$
\left\|Y^{*} h_{\lambda}\right\| \asymp\left\|h_{\lambda}\right\|=\frac{1}{\left(1-|\lambda|^{2}\right)^{1 / 2}} \quad(\lambda \in \mathbb{D}),
$$

where $h_{\lambda}$ are defined in (1.1). 
Proof. By Lemma 2.1, $W$ and $Z$ are quasiaffinities. Therefore, $X$ and $Y$ are quasiaffinities. Intertwining properties of $X$ and $Y$ and the equality $Y X=g_{*}(S)$ easy follow from the construction of $T, X, Y$ and Lemma 2.7.

The power boundedness of $T$ follows from the power boundedness of $R_{0}$ and the equality

$$
\left.P_{B H^{2}} T^{n}\right|_{\mathcal{H}_{0}}=\left.P_{B H^{2}} S^{n}\right|_{\mathcal{K}_{B}} W^{*},
$$

which is a consequence of the relation $Y T=S Y$.

If $\sum_{n \geq 0}|\widehat{g}(n)|<\infty$, then $g_{*}(T)=\sum_{n \geq 0} \overline{\widehat{g}(n)} T^{n}$. Since $Y X Y=g_{*}(S) Y=$ $Y g_{*}(T)$ and $\operatorname{ker} Y=\{0\}$, we conclude that $X Y=g_{*}(T)$.

It is well known that if $R_{0}$ is polynomially bounded, then $\left\{x_{n}\right\}_{n>0}$ is an unconditional basis of $\mathcal{H}_{0}$ (because $\left\{\lambda_{n}\right\}_{n>0}$ satisfies the Carleson condition (2.11), see, for example, [V1, Lemma 2.3]), a contradiction to assumption. Since $R_{0}$ is not polynomially bounded, we conclude that $T$ is not polynomially bounded.

Clearly, $\left\|Y^{*} h_{\lambda}\right\| \leq\|Y\|\left\|h_{\lambda}\right\|$ for every $\lambda \in \mathbb{D}$. Therefore, to prove (2.17), it is sufficient to prove that there exists $c>0$ such that

$$
\left\|Y^{*} h_{\lambda}\right\| \geq c \frac{1}{\left(1-|\lambda|^{2}\right)^{1 / 2}} \text { for every } \lambda \in \mathbb{D}
$$

By $(2.2)$,

$$
\left\|Y^{*} h_{\lambda}\right\|^{2}=\left\|P_{B H^{2}} h_{\lambda}\right\|^{2}+\left\|W P_{\mathcal{K}_{B}} h_{\lambda}\right\|^{2}=|B(\lambda)|^{2}\left\|h_{\lambda}\right\|^{2}+\left\|W h_{B, \lambda}\right\|^{2} .
$$

Let $\left\{D_{n}\right\}_{n \geq 0}$ be defined as in Lemma 2.6. By Lemma 2.6, there exists $c>0$ such that

$$
\left\|W h_{B, \lambda}\right\| \geq \frac{c}{\left(1-|\lambda|^{2}\right)^{1 / 2}} \quad \text { for every } \lambda \in \cup_{n \geq 0} D_{n} .
$$

Let $\lambda \in \mathbb{D} \backslash \cup_{n} D_{n}$. By $(2.15),|B(\lambda)| \geq \delta^{2} / 6$ for such $\lambda$. Therefore,

$$
\left\|Y^{*} h_{\lambda}\right\| \geq \frac{\delta^{2}}{6}\left\|h_{\lambda}\right\| \quad \text { for every } \lambda \in \mathbb{D} \backslash \cup_{n} D_{n} .
$$

Estimate (2.18) is proved.

Remark 2.10. If in Theorem 2.9 one assume that $\left\{x_{n}\right\}_{n \geq 0}$ is a Riezs basis instead of $i s$ not, then $R_{0}$ is similar to a contraction. Therefore, $T$ is similar to a contraction by [C, Corollary 4.2] and $T \approx S$ by [U, Theorem 3.8].

Remark 2.11. A key step in the construction of the example is the existence of a family $\left\{x_{n}\right\}_{n \geq 0}$ satifying (2.7), such that the mapping $W$ acting by the formula $W e_{n}=x_{n}(n \geq 0)$ for an orthonormal basis $\left\{e_{n}\right\}_{n \geq 0}$ is a transformation, but $\left\{x_{n}\right\}_{n \geq 0}$ is not a Riesz basis (Lemma 2.3 and Example 2.4). It seems the basis constructed in [S, Example III.14.5, p. 429] does not have this property.

2.4. Existence of another shift-type invariant subspace. Let $T$ be a polynomially bounded operator on a Hilbert space $\mathcal{H}$ such that $T \prec S$, and let $\mathcal{N} \in \operatorname{Lat} T$ be such that $\left.T\right|_{\mathcal{N}} \approx S$. Then there exists $\mathcal{M} \in \operatorname{Lat} T$ such that $\left.T\right|_{\mathcal{M}} \approx S$ and $\mathcal{N} \vee \mathcal{M}=\mathcal{H}$ (see [G3, Theorem 2.10]). The power bounded operator $T$ on the space $B H^{2} \oplus \mathcal{H}_{0}$ constructed in Theorem 2.9 has the invariant subspace $B H^{2} \oplus\{0\}$ such that $\left.T\right|_{B H^{2} \oplus\{0\}} \cong S$. In this subsection we show that there exists $\mathcal{M} \in$ Lat $T$ such that $\left.T\right|_{\mathcal{M}} \approx S$ and $\left(B H^{2} \oplus\{0\}\right) \vee \mathcal{M}=B H^{2} \oplus \mathcal{H}_{0}$ (although $T$ is not polynomially bounded). 
Lemma 2.12. Suppose that $\mathcal{H}$ is a Hilbert space, $A: \mathcal{H} \rightarrow \mathcal{H}$ is an operator, and $x \in \mathcal{H}$. Then there exist an a.c. contraction $R: \mathcal{K} \rightarrow \mathcal{K}$ and a transformation $X: \mathcal{K} \rightarrow \mathcal{H}$ such that $X R=A X$ and $x \in X \mathcal{K}$ if and only if

there exists a function $w \in L^{1}(\mathbb{T}, m)$ such that $w \geq 0$

$$
\text { and }\|p(A) x\|^{2} \leq \int_{\mathbb{T}}|p|^{2} w \mathrm{~d} m \text { for every (analytic) polynomial } p \text {. }
$$

Proof. "If" part. Denote by $P^{2}(w m)$ the closure of (analytic) polynomials in $L^{2}(w m)$ and by $S_{w m}$ the operator of multiplication by the independent variable on $P^{2}(w m)$. Clearly, $S_{w m}$ is an a.c. contraction. Define the transformation $X: P^{2}(w m) \rightarrow \mathcal{H}$ by the formula $X p=p(A) x$. Then $X 1=x$ and $X S_{w m}=A X$.

"Only if" part. By assumption, there exists $u \in \mathcal{K}$ such that $x=X u$. By [BT, Lemma 3], (2.19) is fulfilled for $R$ and $u$ with some function $w$. Clearly, (2.19) is fulfilled for $A$ and $x$ with the function $\|X\|^{2} w$.

Lemma 2.13. Suppose that $\theta$ is an inner function, $\mathcal{H}_{0}$ is a Hilbert space, $A_{0}: \mathcal{H}_{0} \rightarrow \mathcal{H}_{0}$ is an operator, $Y_{0}: \mathcal{H}_{0} \rightarrow \mathcal{K}_{\theta}$ is a transformation, and $Y_{0} A_{0}=$ $T_{\theta} Y_{0}$. Furthermore, suppose that there exists $x \in \mathcal{H}_{0}$ such that $x$ is a cyclic vector for $A_{0}$ and (2.19) is fulfilled for $A_{0}$ and $x$. Set

$$
A: \theta H^{2} \oplus \mathcal{H}_{0} \rightarrow \theta H^{2} \oplus \mathcal{H}_{0}, \quad A=\left(\begin{array}{cc}
\left.S\right|_{\theta H^{2}} & \left.P_{\theta H^{2}} S\right|_{\mathcal{K}_{\theta}} Y_{0} \\
\mathbb{O} & A_{0}
\end{array}\right) .
$$

Then there exists $\mathcal{M} \in$ Lat $A$ such that $\left.A\right|_{\mathcal{M}} \approx S$ and

$$
\left(\theta H^{2} \oplus\{0\}\right) \vee \mathcal{M}=\theta H^{2} \oplus \mathcal{H}_{0} .
$$

Proof. Set $f=Y_{0} x$. Take $h \in \theta H^{2}$ such that

$$
|h| \geq|f|+w^{1 / 2},
$$

where $w$ is a function from (2.19). Put

$$
\mathcal{M}=\operatorname{clos}\{p(A)(h \oplus x): p \text { is an (analytic) polynomial }\} .
$$

Clearly, $\mathcal{M} \in \operatorname{Lat} A$, and (2.20) is fulfilled, because $x$ is cyclic for $A_{0}$. Furthermore, set $Y=I_{\theta H^{2}} \oplus Y_{0}$ and $\mathcal{E}=\operatorname{clos} Y \mathcal{M}$. Since $Y A=S Y$, we have $\mathcal{E} \in$ Lat $S$, and $\left.\left.Y\right|_{\mathcal{M}} A\right|_{\mathcal{M}}=\left.\left.S\right|_{\mathcal{E}} Y\right|_{\mathcal{M}}$. We will show that

$$
\|Y y\| \geq \frac{\|y\|}{\sqrt{2}} \quad \text { for every } \quad y \in \mathcal{M} .
$$

Then we will obtain that $\left.Y\right|_{\mathcal{M}}$ realizes the relation $\left.\left.A\right|_{\mathcal{M}} \approx S\right|_{\mathcal{E}}$. Since $\left.S\right|_{\mathcal{E}} \cong S$ for every $\mathcal{E} \in$ Lat $S$, the lemma will be proved.

It follows from the relation $Y A=S Y$ that

$$
\begin{aligned}
p(A)(h \oplus x) & =\left(\left.p(S)\right|_{\theta H^{2}} h+\left.P_{\theta H^{2}} p(S)\right|_{\mathcal{K}_{\theta}} Y_{0} x\right) \oplus p\left(A_{0}\right) x \\
& =P_{\theta H^{2}} p(h+f) \oplus p\left(A_{0}\right) x
\end{aligned}
$$

and $Y p(A)(h \oplus x)=p(S) Y(h \oplus x)=p(h+f)$ for every polynomial $p$. 
Therefore,

$$
\begin{aligned}
\| p(A) & (h \oplus x)\left\|^{2}=\right\| P_{\theta H^{2}} p(h+f)\left\|^{2}+\right\| p\left(A_{0}\right) x \|^{2} \\
& \leq\|p(h+f)\|^{2}+\int_{\mathbb{T}}|p|^{2} w \mathrm{~d} m \leq\|p(h+f)\|^{2}+\int_{\mathbb{T}}|p|^{2}|h+f|^{2} \mathrm{~d} m \\
& =2\|p(h+f)\|^{2}=2\|Y p(A)(h \oplus x)\|^{2} .
\end{aligned}
$$

Thus, (2.21) is proved.

Corollary 2.14. Let $T$ be an operator from Theorem 2.9. Then there exists $\mathcal{M} \in$ Lat $T$ such that $\left.T\right|_{\mathcal{M}} \approx S$ and (2.20) is fulfilled (with $\theta=B$ ).

Proof. By Lemma 2.7, $Z^{*} T_{B}=R_{0}^{*} Z^{*}$. Take a vector $u \in \mathcal{K}_{B}$ such $u$ is cyclic for $T_{B}$ and set $x=Z^{*} u$. Since $\operatorname{clos} Z^{*} \mathcal{K}_{B}=\mathcal{H}_{0}, x$ is cyclic for $R_{0}^{*}$. By Lemma $2.12,(2.19)$ is fulfilled for $R_{0}^{*}$ and $x$. Thus, $T$ satisfies to the conditions of Lemma 2.13 with $\theta=B, A_{0}=R_{0}^{*}$ and $Y_{0}=W^{*}$. The conclusion of the theorem follows from Lemma 2.13.

\section{Construction of operators with the Range of arbitrary CODIMENSION}

In this section, for every cardinal number $2 \leq N \leq \infty$ a power bounded operator $T$ is constructed such that $T \prec S$ and $\operatorname{dim} \operatorname{ker} T^{*}=N$. This is impossible for polynomially bounded operators, see Remark 3.3 below. Moreover, for the constructed operator $T$ the estimate $\left\|X^{*} h_{\lambda}\right\| \asymp\left\|h_{\lambda}\right\|$ is fulfilled, where $X$ realizes the relation $T \prec S$ and $h_{\lambda}$ are defined in (1.1).

Lemma 3.1. Suppose that $\mathcal{H}_{0}, \mathcal{E}, \mathcal{K}$ are Hilbert spaces, $T_{0}: \mathcal{H}_{0} \rightarrow \mathcal{H}_{0}$, $A: \mathcal{E} \rightarrow \operatorname{clos} T_{0} \mathcal{H}_{0}, X_{0}: \mathcal{H}_{0} \rightarrow \mathcal{K}, R: \mathcal{K} \rightarrow \mathcal{K}$ are operators and transformations, $X_{0} T_{0}=R X_{0}, X_{0}$ is a quasiaffinity, $R$ is left invertible, $\operatorname{ker} A=\{0\}$ and $A \mathcal{E} \cap T_{0} \mathcal{H}_{0}=\{0\}$. Set

$$
\begin{gathered}
T: \mathcal{H}_{0} \oplus \mathcal{E} \rightarrow \mathcal{H}_{0} \oplus \mathcal{E}, \quad T(x \oplus e)=T_{0} x+A e \quad\left(x \in \mathcal{H}_{0}, e \in \mathcal{E}\right), \\
X: \mathcal{H}_{0} \oplus \mathcal{E} \rightarrow \mathcal{K}, \quad X(x \oplus e)=X_{0} x+v,
\end{gathered}
$$

where $v \in \mathcal{K}$ satisfies the relation $X_{0} A e=R v \quad\left(x \in \mathcal{H}_{0}, e \in \mathcal{E}\right)$.

Then $X T=R X, X$ is a quasiaffinity, $\operatorname{ker} T^{*}=\operatorname{ker} T_{0}^{*} \oplus \mathcal{E}$, and if $T_{0}$ is power bounded, then $T$ is power bounded.

Proof. First, it needs to check that the definition of $X$ is correct, that is, for every $e \in \mathcal{E}$ there exists $v \in \mathcal{K}$ such that $X_{0} A e=R v$ and such $v$ is unique. We have

$$
\begin{aligned}
\operatorname{clos} X_{0} A \mathcal{E} & =\operatorname{clos} X_{0} \operatorname{clos} A \mathcal{E} \subset \operatorname{clos} X_{0} \operatorname{clos} T_{0} \mathcal{H}_{0}=\operatorname{clos} X_{0} T_{0} \mathcal{H}_{0} \\
& =\operatorname{clos} R X_{0} \mathcal{H}_{0}=\operatorname{clos} R \mathcal{K}=R \mathcal{K},
\end{aligned}
$$

the latter equality holds true due to the left invertibility of $R$. We obtain that $X_{0} A \mathcal{E} \subset R \mathcal{K}$. Thus, for every $e \in \mathcal{E}$ the needed $v$ exists, and the uniqueness of $v$ follows from the left invertibility of $R$ again. Furthermore, let $c>0$ be such that $\|R u\| \geq c\|u\|$ for every $u \in \mathcal{K}$. We have

$$
\|X e\|=\|v\| \leq \frac{\|R v\|}{c}=\frac{\left\|X_{0} A e\right\|}{c} \leq \frac{\left\|X_{0} A\right\|}{c}\|e\| \quad \text { for every } e \in \mathcal{E} .
$$


Thus, $X$ is a transformation. The equalities

$$
X T=R X, \quad \operatorname{clos} T\left(\mathcal{H}_{0} \oplus \mathcal{E}\right)=\operatorname{clos} T_{0} \mathcal{H}_{0}, \text { and } \operatorname{clos} X\left(\mathcal{H}_{0} \oplus \mathcal{E}\right)=\mathcal{K}
$$

easy follow from the definitions of $X$ and $T$. The equality for $\operatorname{ker} T^{*}$ is a consequence of the equality for the range of $T$. Let $x \in \mathcal{H}_{0}$, let $e \in \mathcal{E}$, and let $X(x \oplus e)=0$. Then

$0=R X(x \oplus e)=R\left(X_{0} x+v\right)=R X_{0} x+R v=X_{0} T_{0} x+X_{0} A e=X_{0}\left(T_{0} x+A e\right)$.

Since ker $X_{0}=\{0\}$, we conclude that $T_{0} x=-A e$. By assumption, $T_{0} x=$ $-A e=0$. Since $\operatorname{ker} A=\{0\}$, we obtain that $e=0$. From the equalities $X_{0} T_{0}=R X_{0}$, ker $R=\{0\}$, and ker $X_{0}=\{0\}$ we conclude that $\operatorname{ker} T_{0}=\{0\}$. Thus, $x=0$. We obtain that $X$ is a quasiaffinity.

Easy computation shows that

$$
T^{n}(x \oplus e)=T_{0}^{n} x+T_{0}^{n-1} A e \quad \text { for every } n \geq 1, x \in \mathcal{H}_{0}, e \in \mathcal{E} .
$$

Therefore, if $T_{0}$ is power bounded, then $T$ is power bounded.

Corollary 3.2. For every cardinal number $1 \leq N \leq \infty$ there exists a power bounded operator $T$ and a quasiaffinity $X$ such that $X T=S X$, $\operatorname{dim} \operatorname{ker} T^{*}=$ $N$, and

$$
\left\|X^{*} h_{\lambda}\right\| \asymp\left\|h_{\lambda}\right\|=\frac{1}{\left(1-|\lambda|^{2}\right)^{1 / 2}} \quad(\lambda \in \mathbb{D}),
$$

where $h_{\lambda}$ are defined in (1.1).

Proof. In [G2, Theorem 4.4] a cyclic power bounded operator $T_{0}$ and a quasiaffinity $X_{0}$ are constructed such that $X_{0} T_{0}=S X_{0}$,

$$
\left\|X_{0}^{*} h_{\lambda}\right\| \asymp\left\|h_{\lambda}\right\|,
$$

and $T_{0}$ is not left invertible. Since $T_{0}$ is cyclic, we have $\operatorname{dim} \operatorname{ker} T_{0}^{*} \leq 1$. Since $T_{0} \prec S$, we have $\operatorname{dim} \operatorname{ker} T_{0}^{*} \geq 1$. Thus, $\operatorname{dim} \operatorname{ker} T_{0}^{*}=1$, and the corollary is proved for $N=1$.

Let $N \geq 2$. Denote by $\mathcal{H}_{0}$ the space on which $T_{0}$ acts. Set $\mathcal{H}_{1}=\operatorname{clos} T_{0} \mathcal{H}_{0}$. There exists a unitary transformation $U_{1}: \mathcal{H}_{1} \rightarrow \mathcal{H}_{0}$. Set $T_{1}=T_{0} U_{1}$ and consider $T_{1}$ as an operator on $\mathcal{H}_{1}$. Clearly, $T_{1} \mathcal{H}_{1}=T_{0} \mathcal{H}_{0}$. Since $T_{0}$ is not left invertible, $T_{1} \mathcal{H}_{1}$ is not closed. By [FW, Theorem 3.6], there exists a unitary operator $U_{0}$ on $\mathcal{H}_{1}$ such that

$$
T_{1} \mathcal{H}_{1} \cap U_{0} T_{1} \mathcal{H}_{1}=\{0\} .
$$

Take a subspace $\mathcal{E} \subset \mathcal{H}_{1}$ such that $\operatorname{dim} \mathcal{E}=N-1$, if $N<\infty$, or set $\mathcal{E}=\mathcal{H}_{1}$, if $N=\infty$. Put

$$
A=\left.U_{0} T_{1}\right|_{\mathcal{E}} .
$$

Define $T$ as in Lemma 3.1 with $R=S$. Let $X$ be a quasiaffinity from Lemma 3.1. By construction, $P_{\mathcal{H}_{0}} X^{*}=X_{0}^{*}$. Therefore, (3.1) follows from (3.2). Thus, $T$ and $X$ satisfy the conclusion of the corollary.

Remark 3.3. Let $T$ be a polynomially bounded operator, and let $T \prec S$. Then $\operatorname{dim} \operatorname{ker} T^{*}=1$. Indeed, there exists a contraction $R$ such that $R \prec T$ by [BP], and $\operatorname{dim} \operatorname{ker} R^{*}=1$ by [T]. The range of $T$ is closed by [T] and [G1]. 


\section{The essential SPeCtrum of the operator $T_{0}$ From [G2]}

As usually, $\sigma(A), \sigma_{e}(A)$ and $\sigma_{p}(A)$ denote the spectrum, the essential spectrum, and the point spectrum of an operator $A$, respectively. The following lemma is actually proved in [H, Theorem 1].

Lemma 4.1. Let $A$ be a cyclic operator on a Hilbert space such that $\sigma(A) \subset$ clos $\mathbb{D}$ and $A \prec S$. Then $\mathbb{T} \subset \sigma_{e}(A)$ and $\sigma_{e}(A)$ is connected.

Proof. Since $A \prec S$, we have $\mathbb{D} \subset \sigma_{p}\left(A^{*}\right)$. Therefore, $\sigma(A)=\operatorname{clos} \mathbb{D}$ and $\mathbb{T} \subset \sigma_{e}(A)$ by $\left[\mathrm{RR}\right.$, Theorem 0.7]. Since $A$ is cyclic, $\operatorname{dim} \operatorname{ker}\left(A^{*}-\lambda I\right)=1$ for every $\lambda \in \mathbb{D}$. Since $A \prec S$, $\operatorname{ker}(A-\lambda I)=\{0\}$ for every $\lambda \in \mathbb{D}$. We conclude that

$$
\mathbb{D} \backslash \sigma_{e}(A)=\{\lambda \in \mathbb{D}: \operatorname{ind}(A-\lambda I)=-1\} .
$$

By [H, Theorem 1], components of $\mathbb{D} \backslash \sigma_{e}(A)$ are simple connected. Therefore, $\sigma_{e}(A)$ is connected.

We recall the detailed definition of the operator $T_{0}$ used in the previous section.

The operator $T$ from [MT, Remark 2.2] is defined as follows. Let $\left\{e_{j}\right\}_{j \geq 0}$ and $\left\{f_{l j}\right\}_{l \geq 1, j \geq 0}$ be orthonormal bases of Hilbert spaces $\mathcal{E}$ and $\mathcal{F}$, respectively. Put

$$
T f_{l j}=f_{l, j-1}, j \geq 1, \quad T f_{l 0}=0, \quad l \geq 1,
$$

$T e_{j}=e_{j+1}, \quad j \neq 3^{k}, \quad k \geq 1, \quad T e_{3^{k}}=e_{3^{k}+1}+f_{l, 3^{k}}, \quad k \geq 1,2^{l-1} \leq k \leq 2^{l}-1$.

It is proved in [MT] that $T$ is a power bounded operator on $\mathcal{E} \oplus \mathcal{F}$. It is easy to see that $\mathcal{F} \in \operatorname{Lat} T,\left.T\right|_{\mathcal{F}} \cong \oplus_{l \geq 1} S^{*}$, and

$$
\left.P_{\mathcal{E}} T\right|_{\mathcal{E}} \cong S \text {. }
$$

Put

$$
\mathcal{H}_{0}=\bigvee_{n \geq 0} T^{n} e_{0}, \quad T_{0}=\left.T\right|_{\mathcal{H}_{0}}, \quad \text { and } \quad X_{0}=\left.P_{\mathcal{E}}\right|_{\mathcal{H}_{0}}
$$

It is easy to see that $X_{0} T_{0}=\left.P_{\mathcal{E}} T\right|_{\mathcal{E}} X_{0}$ and $\operatorname{clos} X_{0} \mathcal{H}_{0}=\mathcal{E}$. By [G2, Lemma $4.2]$,

$$
\begin{array}{r}
\mathcal{H}_{0}=\left\{u \in \mathcal{E} \oplus \mathcal{F}: u=\sum_{j \geq 0} a_{j} e_{j}+\sum_{l \geq 1} \sum_{j \geq 0}\left(\sum_{k \in \kappa_{l j}} a_{2 \cdot 3^{k}+1-j}\right) f_{l j},\right. \\
\left.\sum_{j \geq 0}\left|a_{j}\right|^{2}+\sum_{l \geq 1} \sum_{j \geq 0}\left|\sum_{k \in \kappa_{l j}} a_{2 \cdot 3^{k}+1-j}\right|^{2}<\infty\right\},
\end{array}
$$

where $\kappa_{l j}=\left\{k \geq 1: j \leq 3^{k}, 2^{l-1} \leq k \leq 2^{l}-1\right\}, l \geq 1, j \geq 0$.

It follows from (4.4) that $\operatorname{ker} X_{0}=\{0\}$. Taking into account the unitarily equivalence (4.2), we can accept that $X_{0}$ realizes the relation $T_{0} \prec S$. It is proved in [G2, Theorem 4.4] that (3.2) is fulfilled and $T_{0}$ is not left invertible.

The following lemma can be easily checked directly, therefore, its proof is omitted. We mention only that if $n=2 \cdot 3^{k}+1-j$ for some $k \geq 1$ and $0 \leq j \leq 3^{k}$, then such $k$ and $j$ are unique. 
Lemma 4.2. Let the Hilbert spaces $\mathcal{E}$ and $\mathcal{F}$ be defined as above, let $\mathcal{H}_{0}$ and $T$ be defined by (4.4) and (4.1), and let $\zeta \in \mathbb{T}$. Define a unitary operator $V_{\zeta}$ on $\mathcal{E} \oplus \mathcal{F}$ by the formulas

$$
\begin{gathered}
V_{\zeta} e_{n}=\zeta^{j} e_{n}, \quad \text { if there exist } k \geq 1 \text { and } 0 \leq j \leq 3^{k} \\
\quad \text { such that } n=2 \cdot 3^{k}+1-j, \\
V_{\zeta} e_{n}=e_{n}, \quad \text { if } n \neq 2 \cdot 3^{k}+1-j \text { for every } k \text { and } j \\
\text { such that } k \geq 1 \text { and } 0 \leq j \leq 3^{k}, \\
V_{\zeta} f_{l j}=\zeta^{j} f_{l j}, \quad j \geq 0, l \geq 1 .
\end{gathered}
$$

Then $\mathcal{F}, \mathcal{H}_{0} \in$ Lat $V_{\zeta}$ and $\left.V_{\zeta} \zeta T\right|_{\mathcal{F}}=\left.T\right|_{\mathcal{F}} V_{\zeta}$.

Theorem 4.3. Let $T_{0}$ be defined by (4.3), and let $\lambda \in \mathbb{D}$. If $\lambda \in \sigma_{e}\left(T_{0}\right)$, then $\{z:|z|=|\lambda|\} \subset \sigma_{e}\left(T_{0}\right)$.

Proof. Let $\lambda \in \sigma_{e}\left(T_{0}\right)$. Then there exists a sequence $\left\{u_{N}\right\}_{N} \subset \mathcal{H}_{0}$ such that $\left\|u_{N}\right\|=1$ for all $N$ and $\lim _{N}\left\|\left(T_{0}-\lambda\right) u_{N}\right\|=0$. We have $u_{N}=x_{N} \oplus y_{N}$, where $x_{N} \in \mathcal{E}$ and $y_{N} \in \mathcal{F}$, and

$$
\left\|\left(T_{0}-\lambda\right) u_{N}\right\|^{2}=\left\|(T-\lambda) y_{N}+P_{\mathcal{F}}(T-\lambda) x_{N}\right\|^{2}+\left\|P_{\mathcal{E}}(T-\lambda) x_{N}\right\|^{2} .
$$

If $\lim \sup _{N}\left\|x_{N}\right\|>0$, then $\lim \sup _{N}\left\|P_{\mathcal{E}}(T-\lambda) x_{N}\right\|>0$ due to (4.2), a contradiction. Thus, $\lim _{N}\left\|x_{N}\right\|=0$. Consequently, $\lim _{N}\left\|(T-\lambda) y_{N}\right\|=0$.

Let $|z|=|\lambda|$. Then $z=\zeta \lambda$ for some $\zeta \in \mathbb{T}$. Let $V_{\zeta}$ be the operator from Lemma 4.2. We have $V_{\zeta} u_{N} \in \mathcal{H}_{0}$ and $\left\|V_{\zeta} u_{N}\right\|=1$ for all $N$. Furthermore, $\left\|\left(T_{0}-z\right) V_{\zeta} u_{N}\right\|=\left\|(T-z) V_{\zeta}\left(x_{N} \oplus y_{N}\right)\right\| \leq\left\|(T-z) V_{\zeta} x_{N}\right\|+\left\|(T-z) V_{\zeta} y_{N}\right\|$.

We have $\lim _{N}\left\|(T-z) V_{\zeta} x_{N}\right\|=0$. By Lemma 4.2,

$$
(T-z) V_{\zeta} y_{N}=V_{\zeta}(\zeta T-z) y_{N}=V_{\zeta} \zeta(T-\lambda) y_{N} \quad \text { for every } N .
$$

We have $\left\|(T-z) V_{\zeta} y_{N}\right\|=\left\|(T-\lambda) y_{N}\right\| \rightarrow 0$. Thus, the sequence $\left\{V_{\zeta} u_{N}\right\}_{N}$ shows that $z \in \sigma_{e}\left(T_{0}\right)$.

Corollary 4.4. Let $T_{0}$ be defined by (4.3). Then $\sigma_{e}\left(T_{0}\right)=\operatorname{clos} \mathbb{D}$.

Proof. By Lemma 4.1, $\sigma_{e}\left(T_{0}\right)$ is connected. If $0 \neq \lambda \in \mathbb{D}$ and $\lambda \notin \sigma_{e}\left(T_{0}\right)$, then, by Theorem 4.3,

$$
\{z:|z|=|\lambda|\} \subset \mathbb{D} \backslash \sigma_{e}\left(T_{0}\right) .
$$

Since the set $\{z:|z|=|\lambda|\}$ is compact and the set $\mathbb{D} \backslash \sigma_{e}\left(T_{0}\right)$ is open, there exists $\varepsilon>0$ such that

$$
\{z:|\lambda|-\varepsilon<|z|<|\lambda|+\varepsilon\} \subset \mathbb{D} \backslash \sigma_{e}\left(T_{0}\right) .
$$

By Lemma 4.1, $\mathbb{T} \subset \sigma_{e}\left(T_{0}\right)$. By [G2, Theorem 4.4], $0 \in \sigma_{e}\left(T_{0}\right)$. Therefore, $\sigma_{e}\left(T_{0}\right)$ can not be connected, a contradiction.

\section{REFERENCES}

[BP] H. Bercovici and B. Prunaru, Quasiaffine transforms of polynomially bounded operators, Arch. Math. (Basel), 71 (1998), 384-387.

[BT] H. Bercovici and K. Takahashi, On the reflexivity of contractions on Hilbert spaces, J. London Math. Soc. (2), 32 (1985), 149-156. 
[BS] N. Borovykh and M. N. Spijker, Bounding partial sums of Fourier series in weighted $L^{2}$-norms, with applications to matrix analysis, J. Comput. Appl. Math., 147 (2002), 349-368.

[C] G. Cassier, Generalized Toeplitz operators, restriction to invariant subspaces and similarity problems. J. Oper. Theory, 53 (2005), No. 1, 4989.

[D] P. L. Duren, Theory of $H^{p}$ spaces, Acad. Press, NY, 1970.

[FW] P. A. Fillmore and J. P. Williams, On operator ranges, Adv. Math., 7 (1971), 254-281.

[G1] M. F. Gamal', On quasisimilarity of polynomially bounded operators, Acta Sci. Math. (Szeged), 81 (2015), 241-249.

[G2] M. F. Gamal', On power bounded operators with holomorphic eigenvectors, Acta Sci. Math. (Szeged), 82 (2016), 545-565.

[G3] M. F. Gamal', Examples of cyclic polynomially bounded operators that are not similar to contractions, II, arXiv:1803.03626.

[H] D. A. Herrero, On multicyclic operators, Integral Equations Oper. Theory, 1 (1978), 57-102.

[L] Yu. Lyubich, Spectral localization, power boundedness and invariant subspaces under Ritt's type condition, Studia Math., 134 (1999), 153167.

[MT] V. Müller and Y. Tomilov, Quasisimilarity of power bounded operators and Blum-Hanson property, J. Funct. Anal., 246 (2007), 385-399.

[NZ] B. Nagy and J. Zemanek, A resolvent condition implying power boundedness, Studia Math., 134 (1999), 143-151.

[N1] N. K. Nikol'skii, Treatise on the shift operator. Springer, Berlin, 1986.

[N2] N. K. Nikolski, Operators, functions, and systems: an easy reading. Volume I: Hardy, Hankel, and Toeplitz, Volume II: Model operators and systems, Math. Surveys and Monographs 93, AMS, 2002.

[RR] H. Radjavi and P. Rosenthal, Invariant subspaces, Springer-Verlag, New York-Heidelberg, 1973.

[S] I. Singer, Bases in Banach spaces. I. Berlin-Heidelberg-New York: Springer-Verlag VIII, 1970.

[T] K. Takahashi, On quasiaffine transforms of unilateral shifts, Proc. Amer. Math. Soc., 100 (1987), 683-687.

[U] M. Uchiyama, Curvatures and similarity of operators with holomorphic eigenvectors, Trans. Amer. Math. Soc., 319 (1990), 405-415.

[V1] P. Vitse, Functional calculus under the Tadmor-Ritt condition, and free interpolation by polynomials of a given degree, J. Funct. Anal., 210 (2004), 43-72.

[V2] P. Vitse, The Riesz turndown collar theorem giving an asymptotic estimate of the powers of an operator under the Ritt condition. Rend. Circ. Mat. Palermo (2), 53 (2004), 283-312.

St. Petersburg Branch, V. A. Steklov Institute of Mathematics, Russian Academy of Sciences, Fontanka 27, St. Petersburg, 191023, Russia

E-mail address: gamal@pdmi.ras.ru 